\title{
Linx
}

Revue des linguistes de l'université Paris X Nanterre

$80 \mid 2020$

L'héritage de Jean Dubois et Françoise Dubois-Charlier

\section{En hommage à Jean Dubois}

\section{Danielle Leeman}

\section{OpenEdition}

\section{Journals}

Édition électronique

URL : http://journals.openedition.org/linx/6626

ISSN : 2118-9692

\section{Éditeur}

Presses universitaires de Paris Nanterre

Référence électronique

Danielle Leeman, «En hommage à Jean Dubois », Linx [En ligne], 80 | 2020, mis en ligne le 31 juillet 2020, consulté le 05 août 2020. URL : http://journals.openedition.org/linx/6626

Ce document a été généré automatiquement le 5 août 2020

Département de Sciences du langage, Université Paris Ouest 


\title{
En hommage à Jean Dubois
}

\author{
Danielle Leeman
}

1 L'image me revient de Jean et Françoise la première fois que je les ai vus ensemble, dans un couloir à Vincennes, l'un à côté de l'autre tout à fait naturellement, comme si de rien n'était, mais tels qu'on était ébloui à les deviner aussi amoureux l'un de l'autre. Pour une étudiante de Jean Dubois, se ressentir ainsi brutalement certaine que son prof était amoureux était totalement imprévisible, de l'ordre de la révélation : l'idée même qu'un professeur pouvait être amoureux était proprement inconcevable. Cette profonde complicité ne se démentira jamais, trouvant son épanouissement aussi dans le travail, dont tous deux avaient la même passion tout en étant complémentaires : d'un côté la culture classique (le grec, le latin, l'histoire du français...), de l'autre la maitrise de la modernité (l'anglais, les Américains, l'informatique...). Jean et Françoise ont beaucoup écrit en commun, et c'est ensemble aussi qu'ils ont construit l'énorme œuvre, malheureusement inachevée, inventoriant les unités de la langue française - œuvre qui ne pouvait que rester inachevée : Françoise m'a écrit, six mois après la mort de Jean, « Vois-tu, la vie a complètement cessé de m'intéresser ». Elle-même disparut dans les trois mois qui suivirent.

2 Jean Dubois était un homme rigoureux et exigeant dans le travail, pour les autres comme pour lui-même. Il possédait, de fait, une capacité incroyable à couvrir un champ empirique vaste - sinon exhaustif, comme c'est le cas pour ses travaux de ces trente dernières années - et à l'analyser finement, méthodiquement, jusqu'au bout. C'est ainsi qu'il a impressionné le neurologue Henri Hécaen et dirigé des thèses en neurolinguistique qui font encore autorité, comme celle de Luce Irigaray (Marcie 1996). Sa passion pour les dictionnaires en est d'ailleurs un autre témoignage par excellence. Donc, oui, Jean Dubois était rigoureux et exigeant dans la recherche - en quoi Françoise et lui se sont bien trouvés! Mais l'homme, comme l'enseignant, n'en était pas moins simple et plein d'humour ${ }^{1}$ - il excellait surtout à faire rire de situations comiques : je ne l'ai jamais entendu se moquer méchamment de tel ou telle collègue.

3 C'est ainsi qu'il racontait très simplement - mais plaisamment - comment, précepteur chez les Lévis-Mirepoix, il n'en mangeait pas moins à la cuisine avec les (autres, précisait-il) domestiques, ou comment, le jour de l'incendie qui a ravagé le château, la 
vieille duchesse complètement myope croyait à un feu d'artifice organisé en l'honneur de son anniversaire (elle n'était peut-être pas seulement myope). Mais Jean Dubois, c'était aussi le professeur qui, issu d'un milieu modeste, s'était inscrit au Parti communiste et en défendait concrètement les valeurs humanistes, en particulier en s'efforçant de fournir de quoi gagner quelque argent à certains étudiants qu'il savait en difficulté, soit par des charges de cours, soit par des piges chez Larousse pour la rédaction de dictionnaires, soit à Sainte-Anne par des travaux de transcription. Dans le même ordre d'idées, il trouvait normal, étant professeur, d'assurer les corvées administratives plutôt que d'en charger les assistants ou les maitres-assistants ${ }^{2}$ pour que ceux-ci puissent consacrer leur temps à la recherche et donc progresser dans leur carrière ; il fut d'ailleurs plutôt surpris dans le milieu des années soixante-dix de voir réclamer par ces collègues l'égalité de traitement dans l'exercice des responsabilités : surpris mais très content de tout ce temps libéré qu'il allait pouvoir consacrer à des travaux autrement plus intéressants!

Car certes, Jean Dubois s'était beaucoup investi dans l'administration, dans la représentation dans les différentes instances, et donc dans les innombrables heures consacrées à des réunions pour les réformes, les programmes, la gestion des carrières, le militantisme syndical - et il y était fort efficace - mais en fait ce qui l'intéressait d'abord et par-dessus tout, c'était le travail intellectuel, la recherche, la découverte - le travail scientifique avec ce qu'il représente d'accumulation minutieuse des données, d'exigence dans l'observation, de rigueur et de clarté dans les conclusions, ce dont on peut voir les résultats tangibles non seulement dans ses publications, mais aussi dans certains jugements portés sur lui. Nicolas Ruwet - avec qui je déjeunais souvent les jours de réunion du Comité de rédaction de Langue française, me disait que Jean Dubois était " génial à l'oral mais moins bon à l'écrit sauf pour le court ", visant ainsi ses Grammaires structurales : ses articles en revanche, synthétiques mais lumineux, étaient un genre qui lui convenait parfaitement.

Maurice Gross, qui travaillait avec Jean Dubois au LADL ${ }^{3}$ avec toute son équipe - j'ai participé à nombre de ces séminaires, qui ont préparé beaucoup de descriptions lexicographiques - appréciait de même ses qualités humaines et scientifiques quoique tous deux n'appartiennent pas à la même génération ni ne relèvent des mêmes formations. Mais, outre leur estime réciproque, leur profonde complicité reposait sans doute aussi sur leur commun intérêt admiratif pour la théorie harrissienne et sa rigueur mathématique. Au moment où je préparais mon numéro de Langages sur $L a$ paraphrase, et où je cherchais qui pouvait traduire une partie de l'article « Report and Paraphrase », Maurice me dit avec ce sourire irrésistible qui le caractérisait : «Dubois est l'une des rares personnes à avoir lu et traduit Harris, tu peux lui demander ?» - estil utile de préciser que je n'aurais jamais osé une chose pareille !

6 Mais si Jean Dubois a très tôt maîtrisé les travaux de Harris, cela ne l'empêche pas de tester rapidement sur le français l'hypothèse chomskyenne, en collaboration avec Françoise Dubois-Charlier : Chomsky publie en 1965 Aspects of the Theory of Syntax et son application systématique à la langue française est publiée dès 1970. Et de même Jean Dubois définit une analyse du discours à partir des données méthodologiques harrisiennes, mais en y intégrant en particulier l'apport benvenistien sur l'énonciation, définissant de la sorte un cadre théorico-descriptif nouveau. Est-il également besoin de rappeler qu'il est le premier à renouveler une tradition lexicographique jusqu'à lui fondée sur des entrées se succédant selon l'ordre alphabétique (ignorant leurs liens 
morphologiques), et des définitions sémantiques classées diachroniquement, illustrées par des définitions littéraires.

7 Jean Dubois avait été recruté par le CNRS pour faire sa thèse d'Etat ; il a créé l'Analyse du discours «à la française » (Maingueneau 1996) et la Neurolinguistique avec Hécaen (Marcie, op. cit.); il a lancé une analyse critique des discours et pratiques pédagogiques justifiant de nouveaux manuels scolaires (Marchand 1996) ; il est considéré aujourd'hui comme l'un des plus prestigieux représentants du Structuralisme (Dosse 1997) et est le «maître à penser» de bon nombre d'ouvrages lexicographiques (Kannas 1996, Rey 2019). Contrairement à la plupart des Professeurs, il ne chercha pourtant pas à prolonger son temps d'université une fois que l'heure de la retraite eut sonné : on allait enfin pouvoir travailler tranquille! C'est que l'ambition n'était pas nulle, mais à la mesure de la passion linguistique du couple pour le lexique appréhendé à travers ses propriétés syntaxiques et distributionnelles : l'ambition, c'était l'entier d'une catégorie dont il s'agissait de rendre compte chaque fois - la totalité des suffixes, ou du fonctionnement du nombre, ou des emplois verbaux, sans oublier l'incroyable Dictionnaire électronique des Mots : autant dire la totalité du Lexique.

Les dictionnaires de Jean et Françoise - tout comme le lexique-grammaire de Maurice Gross - sont aujourd'hui reconnus comme constituant des jalons cruciaux pour les Sciences du langage, de la façon que l'ouvrage Systema Naturae (1735-1758) de Carl von Linné a sans aucun doute fixé les méthodes de travail en Biologie, à partir desquelles la théorie de l'évolution a pu naître une centaine d'années plus tard. Nous sommes tout un groupe de chercheurs à être convaincus que ceux qui sauront utiliser les ressources constituées par Jean Dubois et Françoise Dubois-Charlier et continuer leur projet feront, de même, avancer le domaine de la linguistique de manière durable (Silberztein 2010).

\section{BIBLIOGRAPHIE}

Chomsky, N. (1965). Aspects of the Theory of Syntax. MIT Press.

Dosse, F. (1997). History of Structuralism: The Rising Sign, 1945-1967. Minneapolis (MN): University of Minnesota Press.

Dubois, J., Dubois-Charlier, F. (1970). Eléments de linguistique française - syntaxe. Paris : Larousse.

Kannas, Cl. (1996). « Jean Dubois et la lexicographie », in LINX, n 34-35, pp. 21-25.

Leeman, D. (2015). « Jean Dubois », in Langue française, n¹88, pp. 137-139.

Maingueneau, D. (1996). «Jean Dubois et les débuts de l'analyse du discours en France », in LINX $\mathrm{n}^{\circ} 34-35, \mathrm{pp}$ 27-34.

Marcie, P. (1996). Jean Dubois et l'avènement de la neurolinguistique », in LINX n 34-35, pp. 35-44.

Marchand, F. (1996). « Jean Dubois et la pédagogie », in LINX n 34-35, pp. 45-54. 
Rey, A. (2019). « L'hostilité au père a été quelque chose de fondamental », in Le Monde, Dimanche 8 et Lundi 9 septembre 2019, propos recueillis par Sandrine Blanchard.

Silberztein, M. (2010). « La formalisation du dictionnaire LVF avec NooJ et ses applications pour l'analyse automatique de corpus ", in Langages, n 179-180, pp. 221-242.

Swiggers, P. (2000). « Le champ de la morphologie française : bilan des études et perspectives de recherche ", in Modèles linguistiques, $\mathrm{n}^{\circ} 42$, pp. 14-32.

\section{NOTES}

1. Il annonce par exemple en début de cours à ses étudiants : "Cette année, notre sujet sera le verbe » ou, à propos des temps, «le futur a toujours quelque chose de conditionnel » (Leeman 2015).

2. Cette conception était loin d'être le cas général à l'Université.

3. Laboratoire d'Automatique Documentaire et Linguistique.

\section{AUTEUR}

\section{DANIELLE LEEMAN}

Paris Nanterre \& ICAR (CNRS \& ENS / Lyon 2) 